\section{Delayed bilateral spinal anaesthesia}

Norris et al., ${ }^{1}$ described a case of neuraxial blockade during interscalene block. This shares characteristics with a case we reported in which a patient received an interscalene block and a subsequent subdural injection. ${ }^{2}$ We feel that the Norris case could represent a subdural block.

The dural puncture, which Norris et al. hypothesized, could have allowed entry of the local anaesthetic into the subdural space. Subdural injection presents with an unusually extensive block for the volume of drug injected, a longer onset time, a patchy motor and sensory block, and an unpredictable extent of spread. Subdural block often does not cause a sympathetic block as would occur with a cervical subarachnoid injection.

The interscalene block resulted in a brachial plexus block on the right and a dense neuraxial block of the dermatomes of $\mathrm{C} 2$ to $\mathrm{T} 4$ and lumbar segments. The block was described as dense but the phrenic nerve was not blocked (C3-5). The left C6 dermatome was also unblocked which would not be consistent with a subarachnoid block, which is almost invariably equal and bilateral. If this were a subarachnoid block the block would be expected to set up rapidly and the patient to become unconscious. The presence of a post-dural puncture headache does not eliminate the possibility of a subdural injection, since CSF leakage from the dural sleeve is possible.

Although it is impossible to be certain, we feel that this case represents subdural spread of local anaesthetic. Subdural injections have also been reported during lumbar epidural anaesthesia and confirmed by injection of contrast medium via catheters. ${ }^{3-5}$

\section{J A Dilger, \\ Cleveland, Ohio}

\section{REFERENCES}

1 Norris $D$, Klabsen A, Milne B. Delayed bilateral spinal anaesthesia following interscalene brachial plexus block. Can J Anaesth 1996; 43: 303-5.

2 Tetzlaff JE, Toon HJ, Dilger J, Brems J. Subdural anesthesia as a complication of an interscalene brachial plexus block. Reg Anesth 1994; 19: 357-9.

3 Stevens RA, Stanton-Hicks MD. Subdural injection of local anesthetic: a complication of epidural anesthesia. Anesthesiology 1985; 63: 323-6.

4 Hartrick CT, Pither CE, Pai U, Raj PP, Tomsick TA. Subdural migration of an epidural catheter. Anesth Analg 1985; 64: 175-8.

5 Abouleish E, Goldstein M. Migration of an extradural catheter into the subdural space. A case report. $\mathrm{Br} \mathrm{J}$ Anaesth 1986; 58: 1194-7.

\section{Two oxygen delivery devices compared in a modified laryngeal mask airway}

To the Editor:

We compared the performance of two oxygen delivery devices used with a modified laryngeal mask airway (LMA). ${ }^{1}$ We measured end tidal oxygen concentration at three different oxygen flow rates. ${ }^{2,3}$ The devices investigated were the DAR Hygrobac $S$ filter entraining oxygen through the carbon dioxide sampling port and the Intersurgical 1040-001 T-piece system incorporating a $40 \%$ venturi. ${ }^{4}$

The LMA bowl was removed and stem shortened to allow incorporation of the Datex pressure and volume measurement device whilst maintaining the original LMA size 4 length of $16 \mathrm{~cm}$. The two devices were attached to the free end of the LMA stem in turn whilst 10 supine healthy volunteers, wearing a nose clip, breathed through the stem ensuring a good seal with their lips to avoid air from being entrained.

Measurements of end tidal oxygen concentration were made every $15 \mathrm{sec}$ for $80 \mathrm{sec}$. This cycle was repeated for each device at oxygen flow rates 4,7 and $10 \mathrm{~L} \cdot \mathrm{min}^{-1}$. Mean end tidal oxygen concentration after $180 \mathrm{sec}$ for modified filter vs T-piece were $32 \% v s$ $26 \%$ at $4 \mathrm{~L} \cdot \mathrm{min}^{-1}, 41 \%$ vs $26 \%$ at $7 \mathrm{~L} \cdot \mathrm{min}^{-1}$ and $52 \%$ vs $24 \%$ at $10 \mathrm{~L} \cdot \mathrm{min}^{-1}$ (See Figure below.).

Although we used a modified LMA in awake volunteers further work may confirm that using a modified airway filter to entrain oxygen may be both cheaper and more efficient than the venturi T-piece system when delivering oxygen in the recovery room.

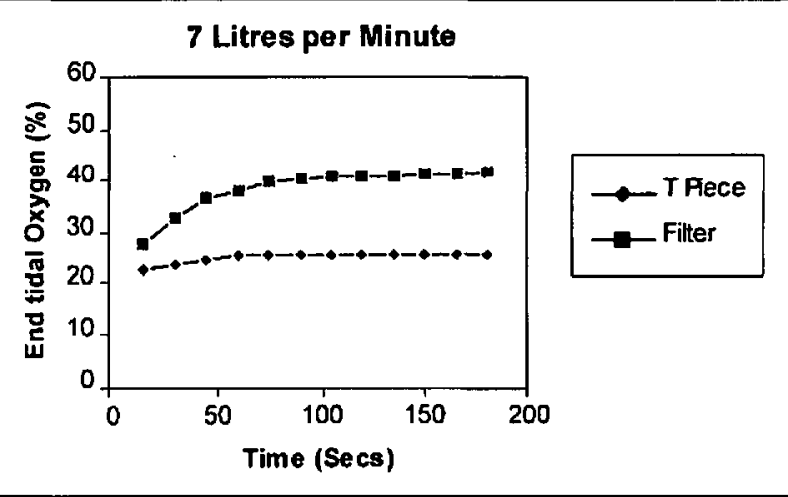

FIGURE Measurements of end tidal oxygen concentration

Adrian K Dashfield FRCA, Jeremy A Langton MD FRCA, Charles G Johnston FFARCSI, Carsten Eickmann DA

Derriford Hospital, Plymouth, England 


\section{RE PERENCES}

1 Broadway PJ, Royle P. Supplemental oxygen and the laryngeal mask airway (Letter). Anaesthesia 1990; 45: 792-3.

2 Myles PS, Heap $M$, Langley $M$. Agreement between end-tidal oxygen concentration and the alveolar gas equation: pre- and postcardiopulmonary bypass. (Abstract) Anaesth Intensive Care 1993; $21: 240$.

3 Berry CB, Myles PS. Preoxygenation in healthy volunteers: a graph of oxygen "washin" using end-tidal oxygraphy. Br J Anaesth 1994; 72: 116-8.

4 Goodwin APL. Postoperative oxygen via the laryngeal mask airway (Letter). Anaesthesia 1991; 46: 700. 